\title{
Human influenza viruses in South-East and East Asia: a systematic review of seasonal patterns, viral types/subtypes, and antiviral susceptibility pattern in the past two decades
}

\author{
Osede Ignis Iribhogbe ${ }^{1 *}$, Sunday Olowo Samuel², John O. Ohaju-Obodo
}

\begin{abstract}
${ }^{1}$ Department of Pharmacology and Therapeutics, ${ }^{2}$ Department of Medical Microbiology, College of Medicine, Ambrose Alli University, Ekpoma Edo State, Nigeria

${ }^{3}$ Department of Pharmacology and Therapeutics, College of Health Sciences, Delta State University, Abraka Delta State, Nigeria
\end{abstract}

Received: 07 July 2020

Revised: 09 August 2020

Accepted: 10 August 2020

*Correspondence:

Dr. Iribhogbe Osede Ignis,

Email: osedeignis@gmail.com

Copyright: ( $)$ the author(s), publisher and licensee Medip Academy. This is an open-access article distributed under the terms of the Creative Commons Attribution Non-Commercial License, which permits unrestricted non-commercial use, distribution, and reproduction in any medium, provided the original work is properly cited.

\begin{abstract}
The study aims to evaluate influenza seasonality pattern and identify influenza viral types/subtype as well as their antiviral susceptibility pattern in South-East (SE) and East Asia (E-Asia) with the view of providing useful information for public health action. The study is a systematic review of existing literature involving the use of search engines such as Google Scholar, PubMed and EBSCOHOST. The search was conducted using a multi-stage approach which yielded 66 articles after screening for relevance to the subject using specific inclusion and exclusion criteria. A total of 60 articles were eventually analyzed and the findings were presented in tables and bar charts. The study showed that the influenza virus was more prevalent in the 6-20 years age group (SE: $53.3 \%$ and E-Asia: 44.8\%) and also occurred commonly in the age of 61-90 years age group (SE: $15.6 \%$ and E-Asia: $24.1 \%$ ). Seasonal peaks were reported more in the winter period with influenza A (H1N1) pdm09 $(22.37 \%$; 24.14\%), seasonal influenza A (H1N1) $(6.58 \% ; 17.24 \%)$, A (H3N2) $(11.84 \% ; 17.24 \%)$, and influenza B Victoria/Yamagata $(5.26 \% ; 20.69 \%)$ being the predominant viral type/subtypes. The pathogenic avian influenza strains; AH5N1 (7.89\%) and A (H7N9) (6.90\%) was also reported in SE and E-Asia respectively. Oseltamivir (37.5\%) and peramivir (37.04\%) were the most frequently used anti-influenza agents in SE and E-Asia respectively. However, an H55Y+I436N combined mutation $4(12.5 \%)$ and an H274Y amino acid substitution $5(18.5 \%)$ in the neuraminidase gene of influenza A (H1N1) pdm09 was associated with decreased sensitivity to neuraminidase inhibitors. The study has shown that influenza A (H1N1) pandemic and seasonal strain, A (H3N2), and B Victoria/Yamagata remains the predominant circulating virus in SE and E-Asia. However, the dynamic antigenic and genetic evolution of the virus calls for more frequent surveillance in the region.
\end{abstract}

Keywords: Viral types/subtypes, Seasonality, Antiviral agents, Antiviral susceptibility

\section{INTRODUCTION}

Influenza viruses are respiratory viruses that belong to a family of viruses known as orthomyxoviridae. ${ }^{1,2}$ They have been implicated in seasonal outbreaks, epidemics, and pandemics in the past two decades. ${ }^{1,3}$ Research has shown that nineteen of the genetically distinct types have shown reassortment since the 1918 Spanish flu which ravaged the world and claimed over 20-50 million lives globally. ${ }^{1,4}$ Additionally, six strains of the genetically distinct subtypes are known to have human-to-human transmission, ten are zoonotic avian influenza viruses 
while three are zoonotic swine influenza viruses., ${ }^{1,3,4}$ However, in the past five years, four novel influenza subtypes and three novel strains of the virus have emerged in humans. ${ }^{1-4}$ Although fourteen different HANA combinations are known to cause human infections, studies have shown that Influenza H1N1, H1N2, and H3N2 HA-NA combinations have emerged multiple times since the Spanish flu pandemic that ravaged the world in $1918 .^{1-3}$

Influenza has continued to be a major cause of morbidity and mortality that is difficult to ignore despite the current global COVID-19 pandemic. The World Health Organization estimates that it is responsible for over 500,000 deaths annually and over 1 billion people are infected with the virus. ${ }^{1,4,5}$ Influenza viruses that are commonly implicated in humans include; influenza A (H1N1) pdm 09, influenza A (H3N2), and influenza B which has two strains Victoria and Yamagata. ${ }^{1-3,6-8}$ The virus causes an acute respiratory tract infection (flu) and has an incubation period that ranges from 1-4 days. ${ }^{1,4}$ The duration is quite short compared to the incubation period of the novel coronavirus COVID-19 which spans from 214 days. ${ }^{1,4,9}$ The infection rate of influenza is usually higher in children (20-30\%) than adults (5-10\%) and contributes between 250-500,000 mortality annually from 3-5 million cases that are reported every year. ${ }^{1,4}$

Additionally, the virus traditionally affects the upper respiratory tracts and rarely involves the lower respiratory tract. ${ }^{1-6,10,11}$ This is another clear difference between it and COVID-19. ${ }^{9}$ Susceptible individuals include the very young, the elderly, immunocompromised

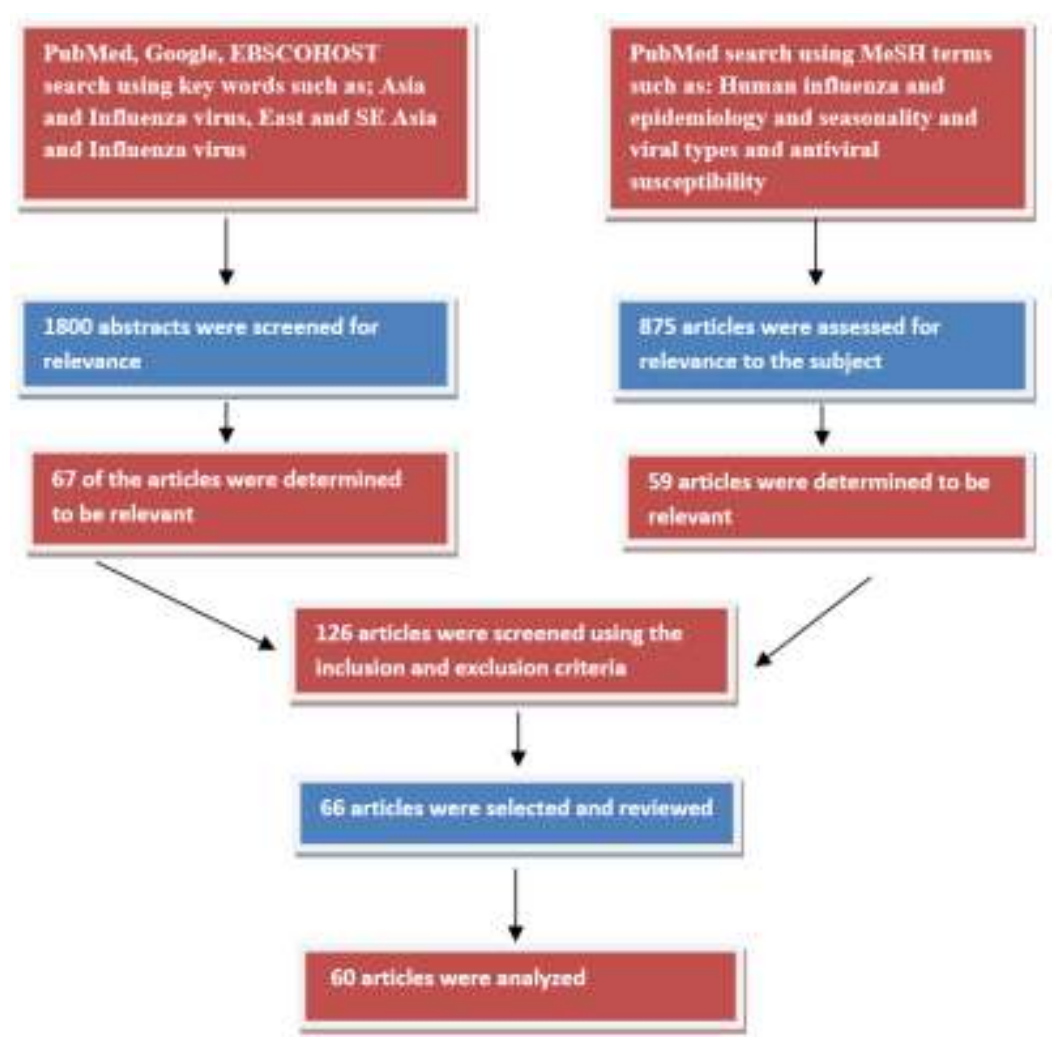

Figure 1: The screening and selection of reviewed articles. individuals, pregnant women, and individuals with predisposing medical conditions such as renal and cardiac impairment. . $^{1,12}$

Additionally, the virus has a diameter of approximately 120 nanometers and has an enveloped segmented RNA genome with antigenic molecules in their nucleocapsid., ${ }^{1,2}$ Antigenic variations usually occur in both the A and B virus with the $C$ virus being more antigenically stable. ${ }^{1-4,6}$ Moreover, the clinical presentation is usually sudden in onset with symptoms ranging from fever, chills, headache, coryza, and dry cough. The duration of fever is usually between 3-5 days with respiratory symptoms lasting for an additional 3-4 days. Symptoms such as cough and weakness persist for up to 2-4 weeks after other symptoms have abated. ${ }^{1,4,13,14}$ In light of this, the review aims to elucidate the seasonal patterns, the viral serotypes, and the antiviral susceptibility pattern of the virus in South-East and East Asia in the past two decades and to identify the public health importance of the seasonal variations in the Asian region.

\section{METHODS}

The study was conducted using search engines such as Google Scholar, PubMed, and EBSCOHOST. In the first stage of the search, keywords such as Asia and Influenza virus, Far East Asia, and influenza virus were used to generate 1,800 abstracts that were screened to identify articles that focused on influenza virus outbreaks and seasonal patterns in both temperate and subtropical areas of Asia. 
In this first stage, 67 articles with a relevant focus on the subject were selected. The second stage of the search involved the generation of abstracts on the subject matter from 16 Asian countries which were grouped into East and South-East Asia respectively. The Asian countries evaluated include Brunei, Cambodia, Indonesia, Laos PDR, Malaysia, Myanmar, Philippines, Singapore, Thailand, Timor Leste, Vietnam, China, Japan, Taiwan, Mongolia, and the Republic of Korea. In the search, keywords such as human influenza and epidemiology and seasonality and country; human influenza type/subtypes and antiviral susceptibility and country (for each specific Asian country) were used in line with standard medical headings (MeSH). These yielded 875 articles that were screened for relevance and later pruned to 66 articles using inclusion and exclusion criteria.

The inclusion criteria include articles that contain information on human influenza virus epidemiology in each of the 16 Asian countries; articles that contain information on human influenza virus seasonality in each of the 16 Asian countries; articles that contain information on human influenza virus subtypes and strains in each of the 16 Asian countries; articles on influenza antiviral susceptibility pattern in each of the 16 Asian countries; articles on human influenza virus that covers the last two decades (1999-2019) in each of the 16 Asian countries.

Exclusion criteria include articles on influenza virus outside the scope of the study; articles that fall short of the inclusion criteria; articles that are not written in the English language.

After reviewing the 66 articles, 60 articles were selected for analysis, and a flow chart showing the schematic analysis was developed based on the PRISMA statement for conducting systematic reviews and meta-analysis as indicated in the website http://prisma-statement.org/ prism statement/checklist.aspx. The analysis was done using IBM-SPSS version 25 and data was presented using tables, proportions, and bar charts.

\section{RESULTS}

\section{Epidemiology, seasonal pattern and influenza viral types/subtypes in past two decades}

Of the 60 articles analyzed 30/60 (50\%) contained information about influenza A virus infection, 20/60 $(33.3 \%)$ contained information about influenza B virus, $9 / 60(15 \%)$ of the reviewed study had information about influenza $A$ and $B$ virus co-transmission while 1/60 $(1.67 \%)$ of the article contained information on influenza $\mathrm{C}$ virus infection which was most prevalent in children.

The mean number of reverse transcriptase PCR (RT-PCR) confirmed cases of Influenza (Table 1) over the past two decades for South-East and East Asia is 94.22 \pm 99.50 and
$1339.83 \pm 3657.63$ respectively. Moreover, the mean influenza virus-related mortality in hospitalized patients in both regions is $10.33 \pm 6.43$ and $303 \pm 362.034$ respectively.

Table 1: Reverse transcriptase-PCR confirmed cases and influenza virus-related mortality in South-East and East Asia.

\begin{tabular}{|ll|}
\hline Region & \multicolumn{1}{l|}{ Mean \pm SD } \\
\hline RT-PCR confirmed cases & \\
\hline South-East Asia $(n=46)$ & $94.22 \pm 99.50$ \\
\hline East Asia $(n=18)$ & $1339.83 \pm 3657.63$ \\
\hline Influenza virus-related mortality \\
\hline South-East Asia $(n=3)$ & $10.33 \pm 6.43$ \\
\hline East Asia $(n=2)$ & $303 \pm 362.04$ \\
\hline
\end{tabular}

Table 2: Age groups commonly predisposed to influenza virus in South-East and East Asia.

\begin{tabular}{|c|c|c|c|}
\hline Region & $\begin{array}{l}\text { Age group } \\
\text { predisposed to } \\
\text { influenza virus }\end{array}$ & Frequency & Percentage \\
\hline \multirow{5}{*}{$\begin{array}{l}\text { South- } \\
\text { East } \\
\text { Asia }\end{array}$} & $1-5$ & 10 & 22.2 \\
\hline & $6-20$ & 24 & 53.3 \\
\hline & $40-60$ & 4 & 8.9 \\
\hline & $61-90$ & 7 & 15.6 \\
\hline & Total & 45 & 100.0 \\
\hline \multirow{5}{*}{$\begin{array}{l}\text { East } \\
\text { Asia }\end{array}$} & $1-5$ & 5 & 17.2 \\
\hline & $6-20$ & 13 & 44.8 \\
\hline & $40-60$ & 4 & 13.8 \\
\hline & $61-90$ & 7 & 24.1 \\
\hline & Total & 29 & 100.0 \\
\hline
\end{tabular}

Also, most influenza virus infections in South-East and East Asia affected the younger age group more predominantly with $53.3 \%$ and $44.8 \%$ occurring in the 620 years age group compared to $15.6 \%$ and $24.1 \%$ in the 61-90 years age groups respectively (Table 2).

Additionally, based on the systematic review the peak period of infection in South-East Asia is usually between June-July (10.53\%). However, 5.26\% of infections spans between January-December yearly. Additionally, most influenza infection in East Asia (Figure 2a and b) occurs between March-April, March-July and October-April $(13.79 \%)$ and can span through October to September $(10.34 \%)$.

Additionally, the influenza type/subtype that circulated predominantly in South-East Asia in the past two decades include influenza A (H1N1) pandemic strain (22.37\%), A (H3N2) (11.84\%), and the co-circulating influenza B Victoria and Yamagata strain (5.26\%). Similarly, in East Asia, influenza A (H1N1) pdm09 (24.14\%), seasonal A (H1N1) (17.24\%), A (H3N2) (17.24\%) and the cocirculating influenza B Victoria and Yamagata strain $(20.69 \%)$ were the most predominant influenza virus 
responsible for respiratory infections (Figure $3 \mathrm{a}$ and $\mathrm{b}$ ). However, co-circulating A (H3N2)/B virus (1.32\%) and influenza $\mathrm{C}$ Victoria/Tokyo $(3.45 \%)$ as well as the pathogenic avian influenza A (H5N1) $(7.85 \%)$ and $\mathrm{A}$ (H7N9) virus $(6.90 \%)$ were also reported in South-East and East Asia respectively during the period under review.
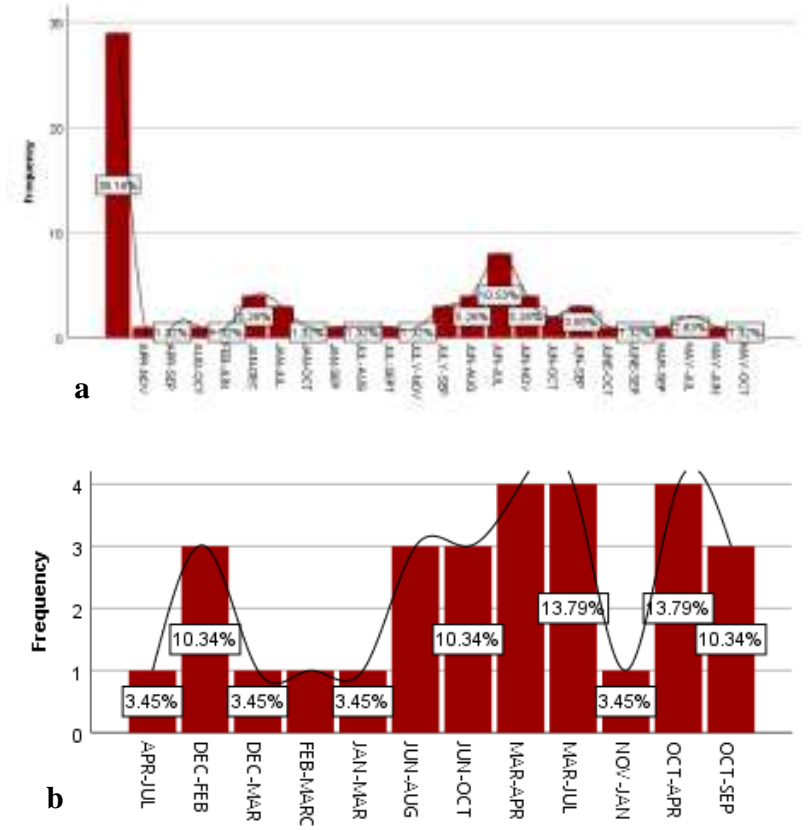

Figure 2: (a) Peak season of viral infection in SouthEast Asia, (b) peak season of viral infection in East Asia.
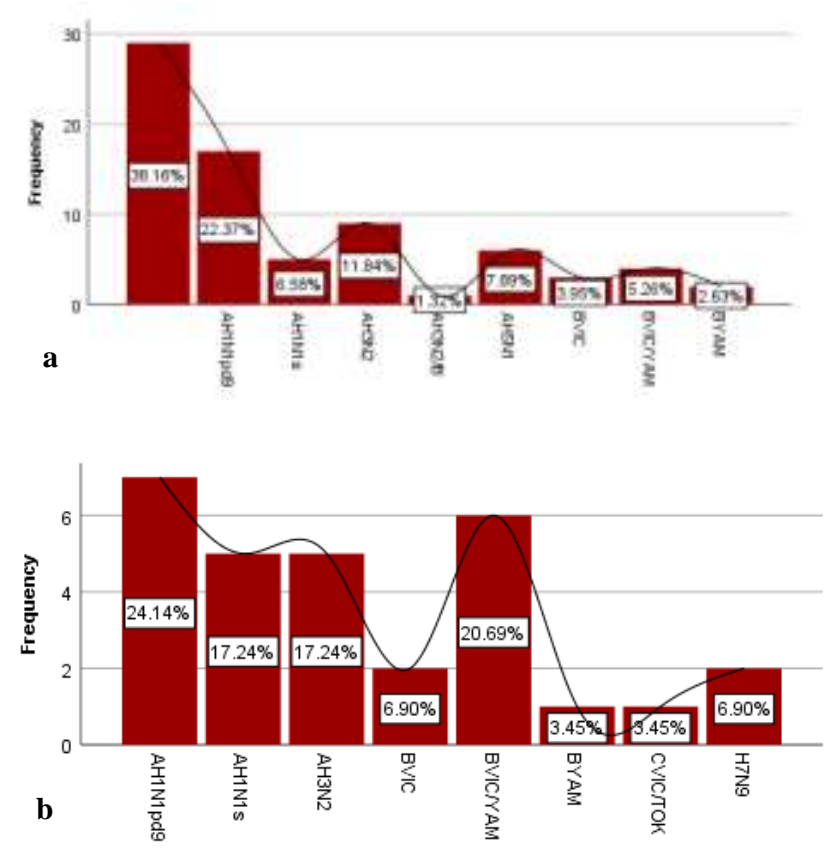

Figure 3: (a) Predominant viral type/subtype in SouthEast Asia in past two decades, (b) predominant viral type/subtype in East Asia in past two decades.
Antiviral susceptibility pattern in past two decades in South-East and East Asia

Findings from the review showed that the neuraminidase inhibitors (NAIs) were the most commonly used drugs for the treatment and prophylaxis of influenza infection in the South-East and East Asia region. Oseltamivir (37.5\%), zanamivir $(21.88 \%)$ and peramivir $(18.75 \%)$ are the most frequently used NAIs in the South-East while peramivir (37.04\%), Oseltamivir (29.63\%) and zanamivir (18.52\%) were the most commonly used NAIs in the eastern region (Figure $4 \mathrm{a}$ and $\mathrm{b}$ ).
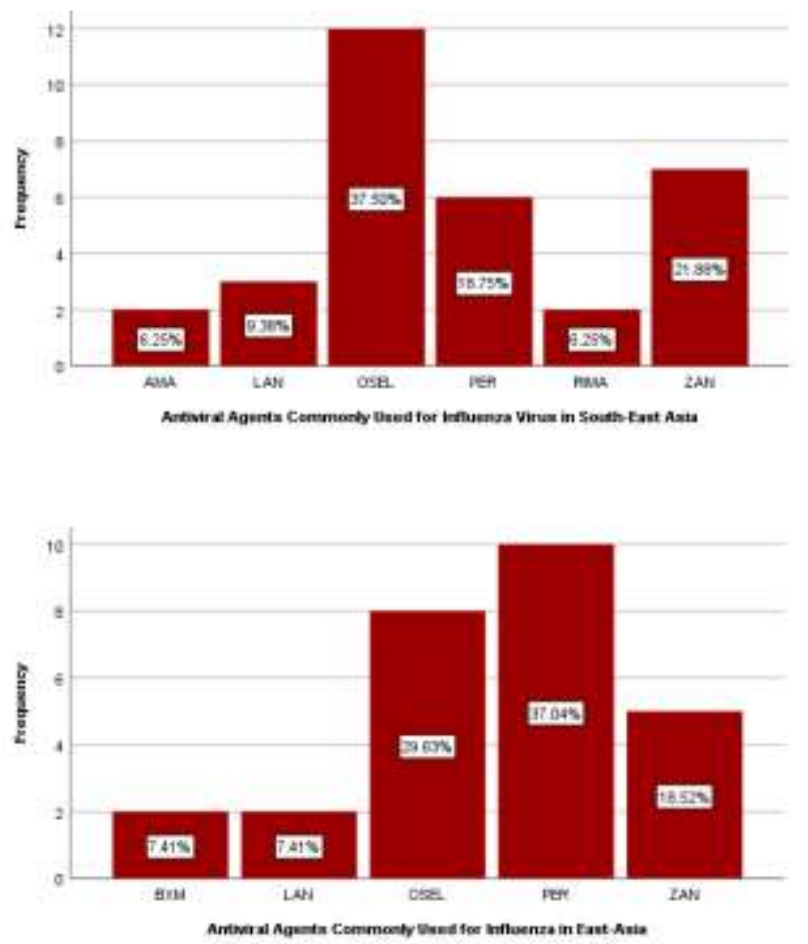

Figure 4: (a) Antiviral agents commonly used for treatment of influenza virus infection in South-East Asia, (b) antiviral agents commonly used for treatment of influenza virus infection in East Asia

BXM=baloxavir marboxil; LAN=laninamivir; OSEL=Oseltamivir; PER=peramivir; ZAN=zanamivir.

Additionally, among the viral types/subtypes reviewed, the neuraminidase gene (NA) was involved in the majority of the mutations associated with a reduction in viral susceptibility in both regions with occasional reports on mutations involving the viral cap-endonuclease polymerase acid protein (PA) in East-Asia. In South-East region the most frequently reported amino acid substitution in the NA gene of influenza A (H1N1) pdm09 includes I436N (12.5\%), combined $\mathrm{H} 275 \mathrm{Y}+\mathrm{I} 436 \mathrm{~N}(12.5 \%)$ and P458T (12.5\%). These mutations caused decreased susceptibility to commonly used NAIs in the region including the most recently introduced NAI laninamivir whose susceptibility is reduced by $\mathrm{H} 275 \mathrm{Y}+\mathrm{I} 436 \mathrm{~N}$ and $\mathrm{P} 458 \mathrm{~T}$ mutation (Table 3 ). 
Table 3: Viral type/subtypes, genetic mutations and antiviral susceptibility in South-East Asia

\begin{tabular}{|c|c|c|c|c|}
\hline $\begin{array}{l}\text { Viral type/ } \\
\text { subtype } \\
\text { (n=32) }\end{array}$ & $\begin{array}{l}\text { Mutations } \\
(\mathbf{n}=32)\end{array}$ & Gene & Antiviral agent & $\begin{array}{l}\text { Antiviral } \\
\text { susceptibility }\end{array}$ \\
\hline \multirow{4}{*}{$\begin{array}{l}\text { A(H1N1) pdm09 } \\
13 \text { (40.6) }\end{array}$} & $\begin{array}{l}\mathbf{N}(\%) \\
\text { I436N } 4(12.5)\end{array}$ & NA & Oseltamivir, Zanamivir & -1 \\
\hline & $\mathrm{H} 275 \mathrm{Y}+\mathrm{I} 436 \mathrm{~N} 4$ (12.5) & NA & $\begin{array}{l}\text { Oseltamivir, Zanamivir, Laninamivir } \\
\text { Peramivir }\end{array}$ & $\begin{array}{l}-1 \\
-1\end{array}$ \\
\hline & D199E $1(3.1)$ & NA & Oseltamivir & -1 \\
\hline & P458T 4 (12.5) & NA & $\begin{array}{l}\text { Oseltamivir, Zanamivir, Laninamivir } \\
\text { Peramivir }\end{array}$ & $\begin{array}{l}-1 \\
-1\end{array}$ \\
\hline \multirow{9}{*}{$\begin{array}{l}\mathrm{A}(\mathrm{H} 5 \mathrm{~N} 1) \\
16(50.0)\end{array}$} & $116 / 117 \mathrm{~N} \mathrm{3(9.4)}$ & NA & Oseltamivir, Zanamivir, Peramivir & +1 \\
\hline & L26I 2(6.3) & M2 & Rimantadine, Amantadine & 0 \\
\hline & S31 2(6.3) & M2 & Rimantadine, Amantadine & +1 \\
\hline & VI149A 1(3.1) & NA & Oseltamivir & -1 \\
\hline & $\mathrm{AI} 3 \mathrm{~V} 1(3.1)$ & NA & Oseltamivir & -1 \\
\hline & $\mathrm{I} 222 \mathrm{~N} 2(6.3)$ & NA & Oseltamivir, Zanamivir & +1 \\
\hline & I117V 1(3.1) & NA & Oseltamivir & -1 \\
\hline & N294S 2(6.3) & NA & Oseltamivir & -1 \\
\hline & $\mathrm{S} 246 \mathrm{~N} 2(6.3)$ & NA & $\begin{array}{l}\text { Oseltamivir } \\
\text { Peramivir }\end{array}$ & $\begin{array}{l}-1 \\
+1\end{array}$ \\
\hline $\begin{array}{l}\text { BVIC/YAM } \\
3(9.4)\end{array}$ & M4261 3(9.4) & NA & Oseltamivir, Zanamivir, Peramivir & -1 \\
\hline
\end{tabular}

NA=Neuraminidase; $-1=$ Decreased drug sensitivity; $0=$ Resistant; $+1=$ Normal drug sensitivity.

Table 4: Viral Type/Subtypes, Genetic Mutations and Antiviral Susceptibility in East-Asia.

\begin{tabular}{|c|c|c|c|c|}
\hline $\begin{array}{l}\text { Viral type/ } \\
\text { subtype } \\
\text { (n=27) } \\
\mathbf{N}(\%)\end{array}$ & $\begin{array}{l}\text { Mutations } \\
(\mathrm{n}=27) \\
\mathbf{N}(\%)\end{array}$ & Gene & Antiviral agents & $\begin{array}{l}\text { Antiviral } \\
\text { susceptibility }\end{array}$ \\
\hline \multirow{5}{*}{$\begin{array}{l}\text { A(H1N1) pdm09 } \\
8(29.6)\end{array}$} & H274Y 5 (18.5) & NA & $\begin{array}{l}\text { Oseltamivir, Peramivir } \\
\text { Zanamivir, Laninamivir }\end{array}$ & $\begin{array}{l}0 \\
+1\end{array}$ \\
\hline & D151G $1(3.7)$ & NA & Oseltamivir & -1 \\
\hline & T148I 1 (3.7) & NA & Zanamivir & -1 \\
\hline & I138T 1 (3.7) & PA & Baloxavir (BXM and BXA) & -1 \\
\hline & H275Y 1 (3.7) & NA & Peramivir & -1 \\
\hline $\begin{array}{l}\text { A(H3N2) } \\
1(3.7)\end{array}$ & I138T 1 (3.7) & PA & Baloxavir (BXM and BXA) & -1 \\
\hline A(H7N9) & H274Y $2(7.4)$ & & Oseltamivir, Zanamivir & -1 \\
\hline $8(29.6)$ & A246T $2(7.4)$ & NA & Oseltamivir, Zanamivir & -1 \\
\hline \multirow{7}{*}{$\begin{array}{l}\text { BVIC/YAM } \\
10(37.0)\end{array}$} & I221T 1 (3.7) & NA & Peramivir & -1 \\
\hline & A245T $1(3.7)$ & NA & Peramivir & -1 \\
\hline & K360E $1(3.7)$ & NA & Peramivir & -1 \\
\hline & A395E $1(3.7)$ & NA & Peramivir & -1 \\
\hline & D4329 $1(3.7)$ & NA & Peramivir & -1 \\
\hline & G145R+Y142H 1 (3.7) & NA & Peramivir & -1 \\
\hline & M4261 4 (14.8) & NA & $\begin{array}{l}\text { Oseltamivir, Zanamivir } \\
\text { Peramivir, Laninamivir }\end{array}$ & $\begin{array}{l}-1 \\
-1\end{array}$ \\
\hline
\end{tabular}

$\mathrm{NA}=$ Neuraminidase; $\mathrm{PA}=$ Polymerase acidic protein; $-1=$ Decreased drug sensitivity; $0=$ Resistant; $+1=$ Normal drug sensitivity; $\mathrm{BXA}=$ Baloxavir acid; $\mathrm{BXM}=$ Baloxavir marboxil (a prodrug of $\mathrm{BXA}$ ).

Additionally, the $116 / 117 \mathrm{~N}(9.4 \%), \mathrm{S} 31(6.3 \%) \mathrm{NA}$ and M2 mutation in influenza A ( H5N1) caused retention of oseltamivir, zanamivir and peramivir sensitivity and the sensitivity of amantadine and rimantadine respectively while the L26I (6.3\%) M2 mutation was associated with the development of resistance to rimantadine and amantadine. Moreover, M4261 (9.4\%) mutation in the NA gene of B Victoria and Yamagata lineage resulted in reduced sensitivity to oseltamivir, zanamivir, and peramivir. 
Similarly, H274Y (18.5\%) NA substitution in the influenza A (H1N1) pdm09 virus was most frequently reported in E-Asia. This, however, was associated with the development of resistance to oseltamivir and peramivir and the retention of sensitivity to zanamivir and laninamivir (Table 4). However, the PA gene amino acid substitution (I138T) in both influenza A (H1N1) pdm09 and influenza A $(\mathrm{H} 3 \mathrm{~N} 2)$ observed in this region was associated with reduced sensitivity to the new drug baloxavir. Additionally, $\mathrm{H} 274 \mathrm{Y}(7.4 \%)$ and A246T (7.4\%) NA substitution in influenza A (H7N9) resulted in reduced sensitivity of the virus to both oseltamivir and zanamivir while I221T, A245T, K360E, A395E, D4329, and combined G145R+Y142H substitution of the NA gene of B Victoria and Yamagata lineage was mainly associated with reduced sensitivity of the virus to peramivir.

\section{DISCUSSION}

\section{Epidemiology, seasonal patterns and viral types/subtypes}

Ever since the first influenza pandemic was reported in the 1580 s, two of the three recent pandemics including the 1918 Spanish flu have originated from Asia which houses $60 \%$ of the world's population. ${ }^{1,3,4,12,14-16}$ The most recent pandemic caused by the novel influenza A virus strain $\mathrm{A}(\mathrm{H} 1 \mathrm{~N} 1)$ pdm09 which started in 2009 originated from Mexico and America and later spread to over 214 countries including South-East and East Asia causing over 18,449 deaths globally. ${ }^{1,3,4,16,17}$ Surveillance data has shown influenza rates of $11-26 \%$ in out-patient and 6$14 \%$ in hospitalized patients in both regions. ${ }^{4}$

However, the majority of Asian countries lie in the northern hemisphere with seasonal influenza patterns varying across the continent and in countries in the southern hemisphere. ${ }^{1,4,18-20}$ In the present review peak influenza infection in South-East Asia occurs in June-July which corresponds with the winter months of JuneAugust in the southern hemisphere. ${ }^{5}$ This is similar to other reports that have shown peak influenza transmission in Asian countries lying in the southern hemisphere during the winter period. ${ }^{18-20}$ Additionally, based on WHO report a country like Malaysia has a year-round transmission similar to what is obtainable in tropical areas like Kenya. ${ }^{21}$ This pattern is different from the seasonal peaks observed in other SE-Asia countries. ${ }^{17,21,22}$ However, a similar all-year-round transmission has been observed in other countries in the region with a tropical and sub-tropical climate. ${ }^{21,22}$ Moreover, in the review, peak influenza infection in East-Asia occurred between March-July which also corresponds with the winter season in the southern hemisphere. This is supported by other researchers that reported months of April-September as the peak period of infection in this region. ${ }^{22,23}$

Moreover, the 9 hemispheric influenza zones developed by the WHO is based on the grouping of countries with similar seasonal patterns and with similar predominant influenza types/subtypes and are usually being reviewed biannually every February for temperate countries in the southern hemisphere and every September for temperate countries in the northern hemisphere. ${ }^{22}$ This is usually based on reports from local sero-surveillance of circulating influenza viruses. ${ }^{20}$ However, the peak period reported in this review is in agreement with the report of Newman et al. ${ }^{22}$ that showed increased influenza activity peaks between November and March in countries in the temperate northern hemisphere and between April and September in countries in the temperate southern hemisphere.

Additionally, the observation that infection predominantly affects the young and the elderly age group in both regions is in agreement with other reports that have shown increased susceptibility in young individuals, the elderly, pregnant women, and in those with chronic health conditions. $^{24-26}$ This has informed the WHO decision to capture this susceptible population in Influenza vaccination programs. ${ }^{27-30}$ Moreover, the viral types reported in this study show a predominance of influenza A (H1N1) seasonal and pandemic strain, A (H3N2), and the B Victoria/Yamagata strain in both regions. However, the highly pathogenic influenza A (H5N1) and A (H7N9) were the zoonotic avian influenza A strain predominantly reported in South-East and East Asia respectively. In other reviews, influenza A (H1N1), A (H3N2), B Victoria/Yamagata, and the zoonotic avian influenza virus A (H5N1) and A (H7N9) was also the predominant influenza virus reported in the region in the past 1-2 decades. . $^{31-34}$

Additionally, based on a very recent WHO report after testing 217, 327 specimens from 60 countries in May 2020,103 of the samples were found to be positive for the Influenza virus of which influenza $\mathrm{A}$ and $\mathrm{B}$ represented $68.9 \%$ and $31.1 \%$ of the positive samples respectively. Also, of influenza, A subtypes A (H1N1) pdm09 (66.7\%) and $\mathrm{A}(\mathrm{H} 3 \mathrm{~N} 2)(33.3 \%)$ were the most dominant subtypes in circulation while B Victoria (100\%) was the predominant influenza $\mathrm{B}$ virus in circulation. ${ }^{30}$ This corroborates the finding of the systematic review. However, the antigenic and genetic evolutionary dynamics of the virus have resulted in the emergence of several subtypes and clades which have been quit diverse across the region. ${ }^{35}$ For instance, there are reports of the emergence of the new clade 1.1.2 and 2.3.2.1 reassortant $\mathrm{A}(\mathrm{H} 5 \mathrm{~N} 1)$ virus and clade $3 \mathrm{c} .2 \mathrm{a}$ and $3 \mathrm{c} .3 \mathrm{a} \mathrm{A}(\mathrm{H} 3 \mathrm{~N} 2)$ virus between 2012-2015. ${ }^{15}, 17$ Within this same period, other new reassortant clades were detected for influenza $A$ (H3N2) and B viruses in Laos PDR, Taiwan, Malaysia, Myanmar, Thailand, Japan, China and Vietnam. ${ }^{35,36}$

The finding implies that there is the need for a sustained approach in the regions periodic update of information provided to the WHO global influenza surveillance response (GISRS) system which is a mechanism designed by the WHO for deciding the antigenic and genetic composition of influenza vaccines used in the different 
hemispheric zones. ${ }^{5,21,27}$ Currently, WHO has approved the use of trivalent or quadrivalent vaccines that protect against the three predominant strains which are; influenza A (H1N1), A (H3N2), and B viruses in most countries. ${ }^{5}$ However, the problem of timing of the vaccines due to variable seasonality and matching of the vaccine components with the prevailing viral type/subtypes due to the rapid evolutionary dynamics of the virus has remained a major challenge globally and in Asia. ${ }^{19,21,22}$ Hence, to stem this challenge countries are encouraged to conduct frequent molecular epidemiological surveillance of these viruses and share the information with the WHO GISRS. ${ }^{18,19}$

Additionally, due to the multiple peaks and residual allyear-round transmission in some of the countries particularly those that lie in the tropical zone in the region, WHO has recommended the administration of seasonal influenza vaccine in periods preceding the primary peak of infection. ${ }^{5,18,19}$ The most recent vaccine recommended for the 2019-2020 influenza season in the northern hemisphere is a quadrivalent vaccine made up of A (H1N1) pdm09-like virus, $\mathrm{A}(\mathrm{H} 3 \mathrm{~N} 2)$-like virus, $\mathrm{B} / \mathrm{Victoria} / 2 / 87$ lineage and B/Yamagata/16/88 lineage while that of the southern hemispheric zone is made up of A/Brisbane/02/2018 (H1N1) pdm09-like virus, A/South Australia/34/2019 A(H3N2)-like virus, B/Washington/ 02/2019-like virus (B Victoria) and B/Phuket/3073/2013like virus (B Yamagata). 5, 28, 29, 37

\section{Antiviral susceptibility pattern}

Moreover, antiviral susceptibility pattern has also been quite diverse in both regions. This is as a result of amino acid substitution involving the neuraminidase protein, the M2 ion channel, and the cap-endonuclease polymerase protein of the virus. ${ }^{38}$ In this review, over $90 \%$ of the mutation was noted to have occurred in the NA gene of the virus which has also been shown in other studies. ${ }^{38,39}$ Several mutations conferring resistance to the NAIs have been reported among which are; mutations in residue 116 \&117, V149A and A13V, N294S and S246N in the NA gene of A (H5N1), and double mutation of $\mathrm{H} 275 \mathrm{Y}+\mathrm{I} 436 \mathrm{~N}$, and D199E and P458T in NA gene of A (H1N1)pdm09 were mostly reported in the South-East region. ${ }^{38-40}$ However, H274Y and A246T NA substitution conferring reduced susceptibility of avian influenza A (H7N9) to oseltamivir and zanamivir and M4261amino acid substitution conferring decreased sensitivity of the virus to all four NAIs (oseltamivir, zanamivir, peramivir and laninamivir) were mostly observed in East Asia. ${ }^{38,41-43}$

Additionally, the I138T amino acid substitution in the PA gene of influenza A (H1N1) pdm09 and A (H3N2) virus which has been linked to reduced sensitivity of the virus to the recently released drug baloxavir is considered to be a potential marker for the monitoring of viral susceptibility pattern to cap-endonuclease polymerase inhibitors. ${ }^{43}$ Globally, resistance to the adamantanes (amantadine and rimantadine) among Influenza A (H3N2) subtypes was $12.3 \%$ and $0.3 \%$ for $\mathrm{A}(\mathrm{H} 1 \mathrm{~N} 1)$ between 2000-2004 with $73.8 \%, 69.6 \%$ and $22.7 \%$ of the report on adamantane resistance coming from China, Hong Kong, and Taiwan respectively. ${ }^{38,44}$ This was mostly associated with S31 mutation in over $95 \%$ of cases and has increased over the years with almost $100 \%$ of influenza A (H3N2) strains from China, Hong Kong, South Korea, Taiwan, and Japan resistant to the adamantanes. ${ }^{38-44}$ Current evidence has shown that most of the strains of influenza A (H1N1) and A (H3N2) circulating globally are resistant to the adamantanes which act mainly by binding to the M2 ion channel in the viral nucleocapsid resulting in the inhibition of viral replication and release. ${ }^{44-46}$

Before the 2009 influenza A (H1N1) pandemic, the majority of the circulating pre-pandemic A (H1N1) influenza strain in Japan (100\%), South-East Asia (32\%), Oceania (86\%) and South-Africa (100\%) were oseltamivir-resistant. The spread of the oseltamivirresistant virus was majorly linked to the emergence of mutant H274Y amino acid substitution in the NA gene. This pattern however changed after the Influenza A (H1N1) 2009 pandemic with the majority of the A (H1N1) subtype exhibiting sensitivity to the NAIs in the region. ${ }^{38-46}$ However, there are still reports of influenza virus resistance to oseltamivir with cross-resistance to peramivir which may be attributed to the ability of the drug-resistant strain to retain replication and transmission fitness overtime thus, creating the possibility of a global health crisis. ${ }^{45-46}$ Another contributory factor to the persistence of drug-resistant strains in the population includes rapid transmission in immunocompromised individuals, the emergence of novel viral strains, and prolonged exposure to subtherapeutic doses of NAIs. ${ }^{45}$

Although, majority of the available report has shown retention of sensitivity to zanamivir and laninamivir, the use of antiviral combination regimen such as laninamivir + IFN- $\lambda$ has been shown to promote the emergence of resistance to laninamivir and other NAIs. ${ }^{40,42-45}$ Additionally, other combination therapies such as oseltamivir with zanamivir and triple combination of amantadine with oseltamivir with ribavirin have not shown any benefit in terms of the outcome of treatment and prevention of the emergence of drug resistance. ${ }^{47,48}$ Therefore the ray of hope in anti-influenza therapy still lies with the development of novel agents to add to the arsenal of currently available agents. Polymerase inhibitors such as favipiravir and baloxavir are novel agents that have demonstrated remarkable efficacy in the treatment of influenza virus infection however, there are a few reports on the presence of resistant variants to the drugs. ${ }^{43,47,49}$ In the light of this, a recent study has shown that $\mathrm{I} 38 \mathrm{~T}$ mutation in the polymerase acidic gene of influenza A (H3N2) virus conferred reduced susceptibility in infants treated with baloxavir. ${ }^{50}$ Hence, the sustenance of frequent molecular surveillance of influenza viruses and antiviral susceptibility monitoring is needed to address this global challenge. 


\section{Limitation of the study}

Although the review provided the key findings of the study, summaries of reviewed studies were not captured in a tabular fashion. This was done to avoid overcrowding of tables in the main text.

\section{CONCLUSION}

The study has shown that influenza A and B viruses still pose a threat of potential epidemic and pandemics which may emanate from Asia or any other part of the world. This in addition to the presence of a wide array of molecular variability and the ability of the virus to retain its replicative and viral fitness has called for the need to maintain and reinvigorate collaborative effort geared towards the sustenance of regular epidemiological surveillance and antiviral drug-resistance monitoring in the region.

Funding: No funding sources Conflict of interest: None declared

Ethical approval: Not required

\section{REFERENCES}

1. Bui CM, Chughtai AA, Adam DC, McIntyre CR. An Overview of the Epidemiology and Emergence of Influenza A Infection in Humans Over Time. Arch Public Health. 2017;75:15.

2. Neumann G, Chen H, Gao GF, Shu Y, Kawaoka Y. H5N1 Influenza Viruses: Outbreaks and Biological Properties. Cell Resp. 2010;20(1):51-61.

3. Simmerman JM, Uyeki TM, The burden of Influenza in East and South-East Asia: a Review of English Language Literature. Influenza Other Respir Viruses. 2008;2(3):81-92.

4. Rafeek RAM, Divarathna MVM, Noordeen F. History and Current Trend in Influenza Virus Infection with Special Reference to Sri Lanka. Virus Disease. 2017;28(3):225-32.

5. WHO. Influenza. Online Referencing. Available at: https://www.who.int/influenza/vaccine/tropics/vaccin ation. Accessed on 15 June 2020.

6. Young BE, Chen M. Influenza in Temperate and Tropical Asia: A Review of Epidemiology and Vaccinology. Human Vaccines Immunotherape. 2020;1-9.

7. Koh MT, Eg KP, and Loh SS. Hospitalized Malaysian Children with Pandemic (H1N1) 2009 Influenza:Clinical Characteristics, Risk factors for Severe Disease and Comparison with the 2002-2007 Seasonal Influenza. Singapore Med J. 2016:57(2):816.

8. Lucero MG, Inobaya MT, Nillos LT, Tan AG, Arguelles VLF, Dureza CJC, et al. National Influenza Surveillance in the Phillipines from 2006 to 2012: Seasonality and Circulating Strains. BMC Infect Dis. 2016;16:762.
9. Azekawa S, Namkoong H, Mitamura K, Kawaoka Y, Saito F. Co-infection with SARS-CoV-2 and Influenza A Virus. Sci Direct. 2020;20:e00775.

10. Saha S, Chadha M, Mamun AA, Rhaman M, SturmRamirez K, Chittaganpitch $\mathrm{M}$, et al. Influenza Seasonality and Vaccination Timing in Tropical and Subtropical Areas of Southern and South-East Asia. Bull World Health Organ. 2014;92(5):318-30.

11. Dapat C, Saito R, Kyaw Y, Naito M, Hasegawa G, Suzuki Y, et al. Epidemiology of Human Influenza A and B Virus in Myanmer from 2005-2007. Intervirology. 2009;52(6):310-20.

12. Stewart RJ, Ly S, Sar B, Leng V, Heng S, Sim K, et al. Using a Hospital Admission Survey to Estimate the Burden of Influenza-associated Severe Respiratory Infection in the Province of CambodiaMethods Used and Lessons Learned. Influenza Other Respir Viruses, 2018;11(1):104-12.

13. Tan AL, Virk RK, Tambyah PA, Inoue M, Lim EA, Chan K, et al. Surveillance and Clinical Characterization of Influenza in a University Cohort in Singapore. PLoS ONE. 2015;10(3):e0119485.

14. Timmermans A, Melendrez MC, Se Y, Chuang I, Samon N, Uthaimongkol N, et al. Human Sentinel Surveillance of Influenza and Other Respiratory Viral Pathogens in Border Areas of Western Cambodia. PLoS ONE. 2016;11(3):e0152529.

15. Ly S, Horwood P, Chan M, Rith S, Sorn S, Oeung K, et al. Seroprevalence and Transmission of Human Influenza A (H5N1) Virus Before and After Reassortment, Cambodia 2006-2014. Emerg Infect Dis. 2017;23(2):300-3.

16. Long-Hay P, Yamamoto E, Bun S, Savuth T, Buntha S, Sokdaro S, et al. Outbreak Detection of InfluenzaLike Illnesses in Prey Veng Province, Cambodia:a Community-based Surveillance. Nagoya J Med Sci. 2019;81(2):269-80.

17. Snoeck CJ, Ponghsavath V, Luetteke N, Kaufmann S, Sausy A, Samountry B, et al. Etiology of Viral Respiratory Infections in Northern Lao People Democratic Republic. J Med Virol. 2018;90(10):1553-8.

18. Hirve S, Newman LP, Paget J, Azziz-Baumgartner E, Fitzner J, Bhat N, et al. Influenza Seasonality in the Tropics and Subtropics-When to Vaccinate? PLoS ONE. 2016;11(4):e0153003.

19. Alonso WJ, Yu C, Viboud C, Richard SA, SchuckPaim C, Simonsen L, et al. A Global Map of Hemispheric Influenza Vaccine Recommendations Based on Local Patterns of Viral Circulation. Scientific Reports. 2015;5:17214.

20. National Geographic Society. Seasons in Asia. Online Referencing. Available at: https://www.nationalgeographic.org/encyclopedia/sea son. Accessed on 13 June 2020.

21. Guerche-Seblain EC, Caini S, Paget J, Vanhems P, Schellevis F. Epidemiology and Timing of Seasonal Influenza in the Asia-pacific Region, 2010-2017: Implications for Influenza Vaccination Programs. BMC Public Health. 2019;19:331.

22. Newman LP, Bhat N, Fleming JA, Neuzil KM. Global Influenza Seasonality to Inform Country- 
Level Vaccine Program:An Analysis of WHO FluNET Influenza Surveillance Data between 2011 and 2016. PLoS ONE. 2018. 13(2):eo193263.

23. Lim C, Ang LW, Tey SH, James L, Kanagalingam D, Su LL, et al. Influenza A (H1N1) pdm09 Infection in Pregnant and Non-pregnant Women Hospitalized in Singapore, May-December 2009. Public Health. 2015;129(6):769-76.

24. Baggett HC, Chittaganpitch $M$, Thamthitiwat S, Prapasiri P, Naorat S, Sawatwong P, et al. Incidence and Epidemiology of Hospitalized Influenza Cases in Rural Thailand during the Influenza A (H1N1)pdm09 Pandemic, 2009-2010. PLoS ONE. 2012;7(11):e48609.

25. Rungrojcharoenkit K, Kittikraisak W, Ditsungnoen D, Olsen SJ, Suntarattiwong P, Chotpitayasunondh T, et al. Influenza Virus Seroprevalence in a Cohort of Healthy and High-risk Children Enrolled in Infancy, Bangkok Thailand. Int J Infect Dis. 2019;89:21-6.

26. Khuntirat B, Yoon IK, Chittaganpitch M, Krueger WS, Supawat K, Blair PJ, et al. High Rate of Influenza $\mathrm{A}(\mathrm{H} 1 \mathrm{~N} 1)$ pdm09 Infections Among Rural Thai Villagers, 2009-2010. PLoS ONE. 2014;9(9):e106751.

27. Barberis I, Martini M, Iavarone F, Orsi A. Available influenza Vaccine; Immunization Strategies, History and New Tools for Fighting the Disease. J Prev Med Hyg. 2016;57(1):e41-46.

28. Carlson R, Southern Hemisphere's 2020 Influenza Vaccine Changed. Precision Vaccination. Available at: https://www.precisionvaccinations.com/whoannounced-2020-flu-season-vaccine-changes. Accessed on 20 June 2020.

29. Center for Infectious Disease Research and Policy (CIDRAP), Two Strains Changed for the Southern Hemisphere 2020 Flu Vaccine. Influenza Vaccines. Available at: https://www.cidrap.umn.edu/newsperspective/2019/09/two-strains-changed-southernhemisphere-2020-flu-vaccine. Accessed on 20 June 2020.

30. WHO, Influenza update June 2020, Update no 309. Available at: https://www.who.int/influenza/ surveillance_monitoring/updates/latest_update_GIP_ surveillance/en/. Accessed on 20 June 2020.

31. Khamphaphongphane B, Ketmayoon P, Lewis HC, Phonekeo D, Sisouk T, Xayadeth S, et al. Epidemiological and Virological Characteristics of Seasonal and Pandemic Influenza in Lao PDR, 20082010. Influenza Other Respir Viruses. 2013;7(3):30411 .

32. Oong XY, Ng KT, Lam TTY, Pang YK, Chan KG, Hanafi NS, et al. Epidemiology and Evolutionary Dynamics of Influenza B Virus in Malaysia, 20122014. PLoS ONE. 2015;10(8):e0136254.

33. Li Q, Zhou L, Zhou M, Chen Z, Li F, Wu H, et al. Epidemiology of Human Infections with Avian Influenza (H7N9) Virus in China. New Engl J Med. 2014;370:520-32.

34. Han DD, Han CX, Li LY, Wang M, Yang JH, Li M. Epidemiology of Human Infection with Avian Influenza A (H7N9) Virus in China 2013-2017.
Zhonghua Liu Xing Bing Xue Za Zhi. 2018;39(1):446.

35. Htwe KTZ, Dapat C, Shobugawa Y, Odagiri T, Hibino A, Kondo H, et al. Phylogeographic Analysis of Human Influenza A and B Viruses in Myanmer, 2010-2015. PLoS ONE. 2019;14(1):e0210550

36. Sunthronwong $\mathrm{N}$, Klinfeung $\mathrm{S}$, Vishiwattana $\mathrm{P}$, Korkong S, Thongmee T, Vongpunsawad S, et al. Genetic and Antigenic Divergence in the Influenza A (H3N2) Virus Circulating between 2016 and 2017 in Thailand. PLoS ONE. 2017;12(12):e0189511.

37. WHO, Recommended Composition of Influenza Virus Vaccines for Use in the 2019-2020 Northern Hemispheric Influenza Season. Available at: https://www.who.int/influenza/vaccines/virus/recom mendations/2019_20_north/en/. Accessed on 20 June 2020.

38. Garcia V and Aris-Brosou S. Comparative Dynamics and Distribution of Influenza Drug Resistance Acquisition to Protein $\mathrm{M} 2$ and Neuraminidase inhibitors. Mol Biol Evol. 2014;31(2):355-63.

39. Gubareva LV, Besselaar TG, Daniels RS, Fry A, Gregory V, Huang W, et al. Global Update on the Susceptibility of Human Influenza Virus to Neuraminidase Inhibitors, 2015-2016. Antiviral Res. 2017;146:12-20.

40. Hurt AC, Besselaar TG, Daniels RS, Ermetal B, Fry A, Gubareva L, et al. Global Update on the Susceptibility of Human Influenza Virus to Neuraminidase Inhibitors, 2014-2015. Antiviral Res. 2016;132:178-85.

41. Hurt AC, Hardie K, Wilson NJ, Ermetal B, Fry A, Gubareva L, et al. Characterization of Widespread Country Cluster of H275Y Oseltamivir-Resistant A (H1N1) pdm 09 Influenza in Australia. J Infect Dis. 2012;206(2):148-57.

42. Leang SK, Kwok S, Sullivan SG, Maurer-Stroh S, Kelso A, Barr IG, et al. Peramivir and Laninamivir Susceptibility of Circulating Influenza A and B Viruses. Influenza Other Respir Viruses. 2014;8(2):135-9.

43. Nakauchi M, Takashita E, Fujisaki S, Shirakura M, Ogawa R, Morita H, et al. Rapid Detection of an I38T Amino Acid Substitution in Influenza Polymerase Acidic Subunit Associated with Reduced Susceptibility to Baloxavir Marboxil. Influenza Other Respir Viruses. 2020;14(4):436-43.

44. Dong G, Peng C, Luo J, Wang C, Han L, Wu B, et al. Adamantane-Resistant Influenza A Virus in the World (1902-2013): Frequency and Distribution of M2 Gene Mutations. PLoS ONE. 2015;10 (3):e 0119115

45. Adams SE, Lugovtsev VY, Kan A, Bovin NV, Donnelly RP, llyushina. Laninamivir-Interferon $\lambda$ Combination Treatment Promotes Resistance by Influenza A Virus More Rapidly than Laninamivir Alone. Antimicrobial Agents and Chemotherapy. 2020;00301-20.

46. Hussain M, Galvin HD, Haw TY, Nutsford AN, Husain M. Drug Resistance in Influenza A Virus the Epidemiology and Management. Infect Drug Resist. 2017;10:121-34. 
47. Lampejo T. Influenza and Antiviral Resistance an Overview. European J Clin Microbiol and Infect Dis. 2020;39(7):1201-8.

48. Duval X, Werf VS, Blanchon T, Mosnier A, Bouscambert-Duchamp M, Tibi A, et al. Efficacy of Oseltamivir-Zanamivir Combination Compared to Each Monotherapy for Seasonal Influenza: A Randomized Placebo-Controlled Trial. PLoS Med. 2010;7(11):e1000362.

49. Reina J, Reina N. Baloxavir Marboxil: A Potent CapDependent Endonuclease Inhibitor of Influenza Viruses. Rev Esp Quimioter. 2019;32(1):1-5.

50. Takashita E, Ichikawa $M$, Morita H, Ogawa R, Fujisaki S, Shirakura M, et al. Human-to-Human
Transmission of Influenza A (H3N2) Virus with Reduced Susceptibility to Baloxavir, Japan, February 2019. Emerg Infect Dis. 2019;25(11):2108-11.

Cite this article as: Iribhogbe OI, Samuel SO, Ohaju-Obodo JO. Human influenza viruses in SouthEast and East Asia: a systematic review of seasonal patterns, viral types/subtypes, and antiviral susceptibility pattern in the past two decades. Int $\mathbf{J}$ Basic Clin Pharmacol 2020;9:1444-53. 